\title{
Oscillatory magnetic tweezers based on ferromagnetic beads and simple coaxial coils
}

\author{
Xavier Trepat, Mireia Grabulosa, Lara Buscemi, and Fèlix Rico \\ Unitat de Biofísica i Bioenginyeria, Facultat de Medicina, Universitat de Barcelona-IDIBAPS, Casanova \\ 143, 08036 Barcelona, Spain \\ Ben Fabry and Jeffrey J. Fredberg \\ Physiology Program, Harvard School of Public Health, 665 Huntington Avenue, Boston, Massachusetts 02115 \\ Ramon Farréa) \\ Unitat de Biofísica i Bioenginyeria, Facultat de Medicina, Universitat de Barcelona-IDIBAPS, Casanova \\ 143, 08036 Barcelona, Spain
}

(Received 12 March 2003; accepted 6 June 2003)

\begin{abstract}
We report the design and validation of simple magnetic tweezers for oscillating ferromagnetic beads in the piconewton and nanometer scales. The system is based on a single pair of coaxial coils operating in two sequential modes: permanent magnetization of the beads through a large and brief pulse of magnetic field and generation of magnetic gradients to produce uniaxial oscillatory forces. By using this two step method, the magnetic moment of the beads remains constant during measurements. Therefore, the applied force can be computed and varies linearly with the driving signal. No feedback control is required to produce well defined force oscillations over a wide bandwidth. The design of the coils was optimized to obtain high magnetic fields (280 mT) and gradients $(2 \mathrm{~T} / \mathrm{m})$ with high homogeneity $(5 \%$ variation) within the sample. The magnetic tweezers were implemented in an inverted optical microscope with a videomicroscopy-based multiparticle tracking system. The apparatus was validated with $4.5 \mu \mathrm{m}$ magnetite beads obtaining forces up to $\sim 2 \mathrm{pN}$ and subnanometer resolution. The applicability of the device includes microrheology of biopolymer and cell cytoplasm, molecular mechanics, and mechanotransduction in living cells. (C) 2003 American Institute of Physics. [DOI: 10.1063/1.1599062]
\end{abstract}

\section{INTRODUCTION}

The mechanical properties of biological soft samples including biomolecules, ${ }^{1,2}$ biopolymers, ${ }^{3,4}$ and cells ${ }^{5-8}$ can be probed by manipulating magnetic microbeads embedded in or attached to the sample. Like other recently developed micro- and nanomanipulation techniques ${ }^{9}$ such as scanning force microscopy, ${ }^{10}$ optical tweezers,,${ }^{11,12}$ and microneedles, ${ }^{13}$ magnetic tweezers can apply forces in the piconewton scale while sensing the sample response with nanometer resolution. Moreover, when compared with these techniques, magnetic tweezers offer a number of advantages such as the possibility to track a large number of probes simultaneously or the absence of a laser focused on the probe that could damage the sample. ${ }^{14,15}$

Micromanipulation of magnetic beads has been achieved by using either movable permanent magnets ${ }^{1,16}$ or systems based on one or more electromagnets. Permanent magnets have the advantage of simplicity, portability, and no requirement for power. ${ }^{17}$ However, time-varying forces are more easily applied using electromagnets. The most common systems to obtain homogeneous force fields over a wide sample area use a single pair of electromagnets to produce a magnetic gradient. ${ }^{4,18,19}$ The homogeneity and linearity of these systems can be improved by adding a second pair of coils to

${ }^{a)}$ Electronic mail: rfarre@ub.edu superimpose a homogeneous magnetizing field onto the gradient. ${ }^{20}$ More complex devices which apply forces in more than one dimension have been described. For instance, Amblard and co-workers developed a system based on four sets of independent electromagnets normal to the optical axis of a microscope that allowed the rotation and biaxial translation of magnetic beads in a controlled way. ${ }^{21}$ More recently, Gosse and Croquette designed a magnetic setup built with six electromagnets coupled to a video-tracking system to apply vertical and lateral forces. ${ }^{22}$

The abovementioned magnetic tweezers based either on permanent magnets or electromagnets with soft iron cores suffer from a number of limitations. First, the generated magnetic fields and gradients cannot be accurately computed and, consequently, a calibration of the system is required to determine the amplitude and the homogeneity of the force field applied for each type of particle used. Second, the hysteretic effects related to the use of soft magnetic materials demand a feedback loop to control the applied field in a relatively low bandwidth. ${ }^{21}$ Third, as the magnetization of the beads is dependent on the applied magnetic field, their magnetic moment varies during experiments that involve time-varying forces. Moreover, this dependence is nonlinear and, therefore, the force generated on the beads is not proportional to the driving signal. ${ }^{19}$ Fourth, the habitual use of superparamagnetic beads with low magnetic moment requires the generation of large gradients to reach relatively 
low forces. These limitations can be overcome by employing simple coaxial coils and ferromagnetic (or ferrimagnetic) beads. Indeed, the use of coaxial coils avoids bandwidth limitations and allows the computation and optimization of the force field over the sample with exact solution. Moreover, the magnetic moment of ferromagnetic beads can be kept constant and known throughout the measurements.

The aim of this work was to design and validate oscillatory magnetic tweezers based on a single pair of coaxial coils and ferromagnetic beads. The device works in two successive and independent modes: magnetization of the ferromagnetic beads, and application of oscillatory translational forces. In the magnetization mode, the beads are permanently magnetized with a brief and large magnetic pulse. ${ }^{23}$ This is achieved by connecting the coils in direct configuration, i.e., with the same current flowing in the same direction in each coil. In the force mode, an oscillatory magnetic gradient is applied to the sample producing an axial force. This is accomplished by connecting the coils in inverse configuration, i.e., inverting the polarity of one of the coils in such a way that a homogeneous magnetic gradient is generated while the field in the central plane vanishes. ${ }^{24}$ In this mode, a low constant field is superimposed onto the gradient to keep the beads aligned in the axial direction. The magnetic tweezers were implemented in an inverted optical microscope with a videomicroscopy multiparticle tracking system that allowed the detection of oscillatory displacements with subnanometric precision. The system was validated in the $\mathrm{pN}$ and $\mathrm{nm}$ range by embedding ferrimagnetic beads in a liquid of known viscosity.

\section{SYSTEM DESIGN}

\section{A. Coil design}

\section{Principle and design requirements}

In the presence of a magnetic field $\mathbf{B}$, a magnetic dipole with magnetic moment $\mathbf{m}$ senses a force $\mathbf{F}$ given by ${ }^{25}$

$$
\mathbf{F}=\boldsymbol{\nabla}(\mathbf{m} \cdot \mathbf{B}) .
$$

If $\mathbf{m}$ is constant and field aligned in an arbitrary direction $z$, Eq. (1) simplifies to:

$$
F=m \frac{\partial B_{z}}{\partial z} .
$$

Accordingly, the device described in this work was designed to produce forces orthogonal to the optical axis of an inverted optical microscope (Axiovert S100 Zeiss, Gottingen, Germany) through a pair of circular coils (Fig. 1). The main geometric constraints of the setup were the distance between the condenser and the microscope stage $(50 \mathrm{~mm})$ and the width of the objective ( $24 \mathrm{~mm}$, A-plan $40 \times$ Zeiss, Gottingen, Germany) which had to fit between the coils. Accounting for these limitations, the coil dimensions and separation were optimized in accordance with the following conditions: (a) in direct configuration, the magnetizing field $B_{z}$ had to be large enough to magnetize the beads to saturation; (b) in inverse configuration the $\partial B_{z} / \partial z$ component of the gradient had to be as large as possible to maximize the force; (c) in both configurations the homogeneity of the field and of the gradi-

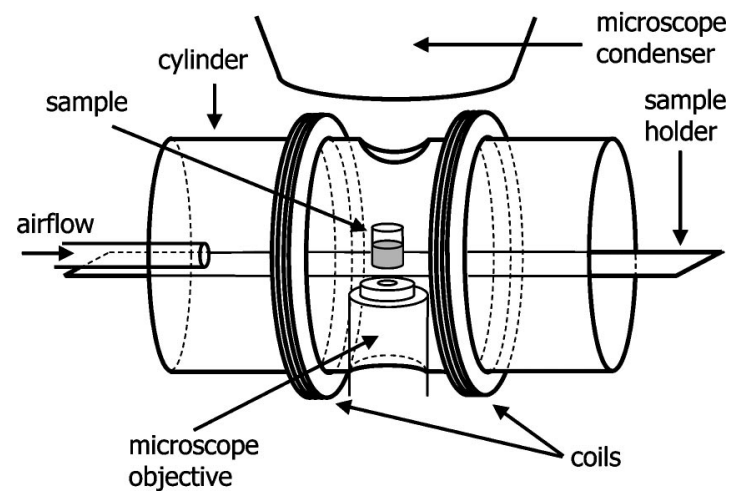

FIG. 1. Schematic view of the magnetic tweezers. The coils (400 turns per coil, $0.5 \mathrm{~mm}$ wire) were wound around a methacrylate cylinder to ensure parallel turns and minimize alignment errors. The sample was enclosed inside the cylinder with two circular apertures to keep the optical axis free. To avoid mechanical coupling, the sample was placed on a methacrylate stage that was introduced inside the tube without contacting the coils structure. Laminar airflow was circulated through the tube to control the sample temperature. The coils were externally cooled by a fan. Both the methacrylate stage and the coils tube were independently mounted on the microscope stage.

ent had to ensure that all the beads in the sample (typically a $7 \mathrm{~mm}$ diameter well) had the same magnetic moment and sensed the same force within a defined accuracy.

\section{On-axis optimization}

The magnetic field at the center of the axis of a pair of circular coaxial coils connected in direct configuration can be expressed as:

$$
B_{z}=\frac{\mu_{0} N I}{d}\left[\frac{\alpha^{2}}{\left(\alpha^{2}+\frac{1}{4}\right)^{3 / 2}}\right],
$$

where $\mu_{0}=4 \pi \times 10^{-7} \mathrm{Tm} / \mathrm{A}$ is the free space magnetic permeability, $N$ is the number of turns of each coil, $I$ is the current in the coils, $d$ is the separation between them and $\alpha$ $=R / d, R$ being the radius of the coils. In inverse configuration, $B_{z}$ vanishes at the midplane between the coils and the axial magnetic gradient is given by:

$$
\frac{\partial B_{z}}{\partial z}=\frac{3 \mu_{0} N I}{2 d^{2}}\left[\frac{\alpha^{2}}{\left(\alpha^{2}+\frac{1}{4}\right)^{5 / 2}}\right] .
$$

The device was optimized by bringing the coils as close as possible, i.e., minimizing $d$, and by maximizing the magnitude and homogeneity of $B_{z}$ and $\partial B_{z} / \partial z$ as a function of the nondimensional parameter $\alpha$. Homogeneity was analyzed from Eqs. (3) and (4) by defining the variation of $B_{z}$ and $\partial B_{z} / \partial z$ as the difference between their central and extreme values expressed as a percentage of their central value. As shown in Fig. 2, the magnitude and homogeneity of $B_{z}$ and of $\partial B_{z} / \partial z$ were maximum for four different values of $\alpha$. The maximum field and gradient were obtained, respectively, at $\alpha=1 / \sqrt{ } 2=0.71$ and $\alpha=1 / \sqrt{ } 6=0.41$. The homogeneity was maximum (the variation was minimum) at $\alpha=1$ for $B_{z}$ in direct configuration and $\alpha=1 / \sqrt{ } 3=0.58$ for $\partial B_{z} / \partial z$ in inverse 


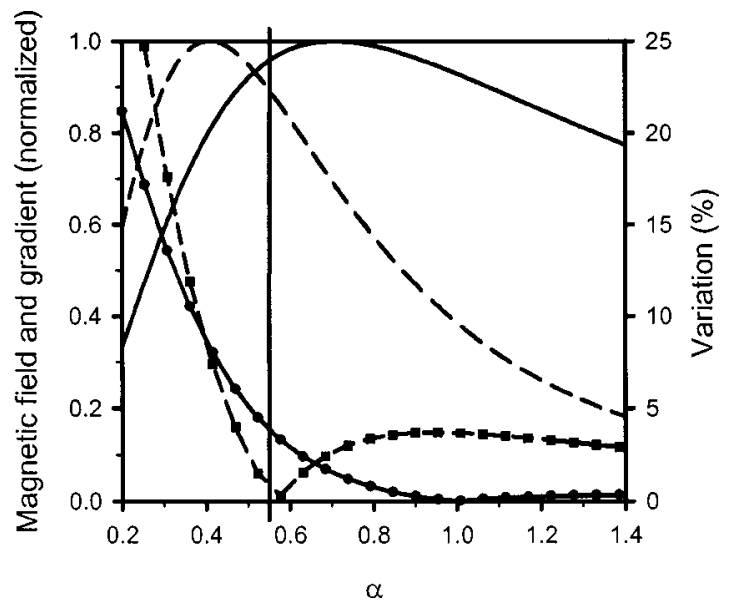

FIG. 2. Four magnetic variables optimized as a function of the parameter $\alpha=R / d$ ( $R$ and $d$ are coil radius and separation, respectively). Left axis: magnetic field $B_{z}$ in direct configuration (solid line, no symbols) and magnetic gradient $\partial B_{z} / \partial z$ in inverse configuration (dashed line, no symbols) evaluated at the center of the coil axis. Right axis: variation of $B_{z}$ in direct configuration (circles) and $\partial B_{z} / \partial z$ in inverse configuration (squares) evaluated over the central area corresponding to the sample location $(7 \mathrm{~mm}$ diameter circle). The vertical line indicates the final value $\alpha=0.56$ chosen for the implementation of the magnetic tweezers.

configuration. These values correspond to those that make the second derivatives of $B_{z}$ and $\partial B_{z} / \partial z$ with respect to $z$ vanish. The final value of $\alpha$ was chosen in such a way that the magnetic field and gradient were larger than $90 \%$ of their maximum values while their variation did not exceed $5 \%$ within the sample along the coil axis: $\alpha=0.56$ with $R$ $=20 \mathrm{~mm}$ and $d=36 \mathrm{~mm}$ (Fig. 2).

\section{Off-axis analysis}

A single pair of coaxial coils exhibits both an axial and a radial component of the magnetic field. When the system is fed by an oscillating current, any magnetic bead in the sample that is not exactly located along the coil axis or in the central plane between the coils senses an oscillating torque $\mathbf{T}$ given by:

$$
\mathbf{T}=\mathbf{m} \times \mathbf{B} .
$$

According to this equation, the bead tends to orient with the time-varying magnetic field. More precisely, the oscillatory axial component of the field $B_{z}$ forces the bead to pivot each time the direction of the field is inverted and the radial component $B_{\rho}$ causes a rotation of the bead on the sample plane.

To evaluate the off-axis components of the field we performed a numerical integration of the Biot and Savart law applied to a pair of circular coaxial coils of radius $R$ and separation $d$. Equations (6), (7), and (8) correspond to the three components of the field at any point in the space with cylindrical coordinates $\rho, \phi$, and $z$, where $z$ is the component in the coils axis direction (see Fig. 3 for notation). The origin of coordinates is located at the center of the left coil:

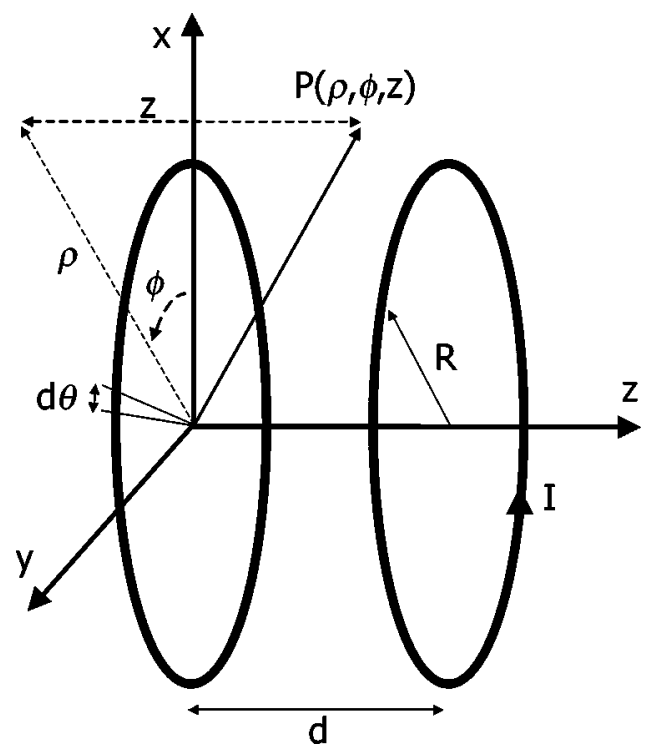

FIG. 3. Cylindrical coordinate system used to compute the magnetic field created by a pair of coaxial coils of radius $R$ and separation $d$ carrying a current $I$. The field was calculated at any point $P$ with coordinates $\rho, \phi$, and $z$. The line element $R d \theta$ was integrated along the wire path for each coil.

$$
\begin{aligned}
B_{\rho}= & \frac{\mu_{0} N I}{4 \pi}\left[\int_{0}^{2 \pi} \frac{z R \cos (\theta) d \theta}{\left[\rho^{2}+R^{2}-2 \rho R \cos (\theta-\phi)+z^{2}\right]^{3 / 2}}\right. \\
& \left. \pm \int_{0}^{2 \pi} \frac{(z-d) R \cos (\theta) d \theta}{\left[\rho^{2}+R^{2}-2 \rho R \cos (\theta-\phi)+(z-d)^{2}\right]^{3 / 2}}\right],
\end{aligned}
$$

$$
\begin{aligned}
B_{\phi}= & \frac{\mu_{0} N I}{4 \pi}\left[\int_{0}^{2 \pi} \frac{z R \sin (\theta) d \theta}{\left[\rho^{2}+R^{2}-2 \rho R \cos (\theta-\phi)+z^{2}\right]^{3 / 2}}\right. \\
& \left. \pm \int_{0}^{2 \pi} \frac{(z-d) R \sin (\theta) d \theta}{\left[\rho^{2}+R^{2}-2 \rho R \cos (\theta-\phi)+(z-d)^{2}\right]^{3 / 2}}\right],
\end{aligned}
$$

$$
\begin{aligned}
B_{z}= & \frac{\mu_{0} N I}{4 \pi}\left[\int_{0}^{2 \pi} \frac{R^{2}-\rho R \cos (\theta-\phi) d \theta}{\left[\rho^{2}+R^{2}-2 \rho R \cos (\theta-\phi)+z^{2}\right]^{3 / 2}}\right. \\
& \left. \pm \int_{0}^{2 \pi} \frac{R^{2}-\rho R \cos (\theta-\phi) d \theta}{\left[\rho^{2}+R^{2}-2 \rho R \cos (\theta-\phi)+(z-d)^{2}\right]^{3 / 2}}\right] .
\end{aligned}
$$

These expressions were obtained from the integration of the field created by a line element $R d \theta$ with coordinates $(R, \theta, 0)$ along the wire path for each coil. The positive sign corresponds to the direct configuration and the negative sign to the inverse configuration. From symmetry considerations, the azimuthal coordinate $B_{\phi}$ is always zero. Figure 4 illustrates the low variation of both $B_{z}(3 \%)$ and $\partial B_{z} / \partial z(1 \%)$ over the sample area, suggesting that an on-axis analysis of the axial component could be extended off-axis with little error for both the direct and the inverse configurations. $B_{\rho}$ vanishes on-axis and increases with the radius according to the second Maxwell equation: 
a.

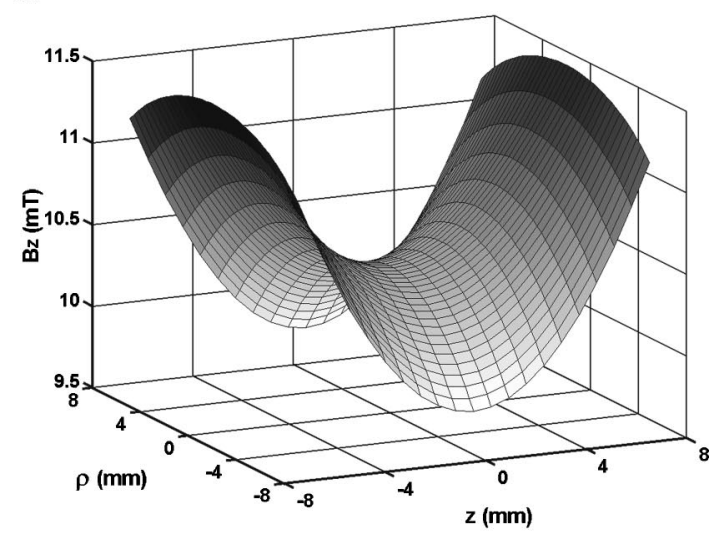

b.

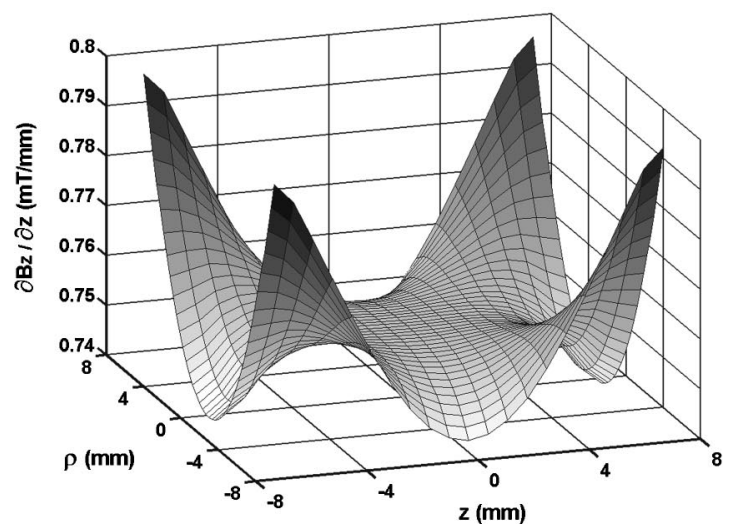

FIG. 4. Numeric computation of the axial component $B_{z}$ of the magnetic field in direct configuration (top) and of the axial component of the magnetic gradient $\partial B_{z} / \partial z$ in inverse configuration (bottom) for a current of $1 \mathrm{~A}$. The numeric integration of the Biot and Savart law was performed on a circle of $14 \mathrm{~mm}$ diameter corresponding to twice the sample diameter.

$$
\frac{1}{\rho} \frac{\partial}{\partial \rho}\left(\rho B_{\rho}\right)+\frac{\partial B_{z}}{\partial z}=0
$$

Integration of this equation shows that $B_{\rho}$ is negligible in direct configuration in such a way that the maximum angle between the coil axis and the magnetic field is $1^{\circ}$ in the sample region. In inverse configuration, integration of Eq. (9) leads to: ${ }^{26}$

$$
\frac{\partial B_{\rho}}{\partial \rho} \approx-\frac{1}{2} \frac{\partial B_{z}}{\partial z}
$$

hence, $\partial B_{\rho} / \partial \rho$ varies linearly with the axial component. The crossed partial derivatives, $\partial B_{\rho} / \partial z$ and $\partial B_{z} / \partial \rho$, are equal and their values are much smaller $(<0.5 \%)$ than $\partial B_{\rho} / \partial \rho$ and $\partial B_{z} / \partial z$ in the sample.

\section{Alignment field}

The field superimposed onto the gradient to keep the beads aligned with the coil axis had to meet the following requirements in a central area corresponding to the microscope field of view $(\sim 1 \mathrm{~mm}$ at $10 \times$ magnification): first, it had to be as large as possible to minimize the rotation of the beads due to the off-axis radial field $B_{\rho}$; second, it had to exceed the maximum axial field $B_{z}$ produced during one oscillation $(1.2 \mathrm{mT})$ to prevent any bead from pivoting; third, it had to remain low enough to avoid a remagnetization of the beads and to restrict the heat dissipation in the coils. Taking into account these requirements, the alignment field was set to $6 \mathrm{mT}$, resulting in a maximum on-plane rotation of $5^{\circ}$ in the central region (1 $\mathrm{mm}$ diameter) of the sample.

\section{B. Current supply and magnetizer}

\section{Computer controlled current supply}

A double-channel voltage-controlled current source was built by connecting each coil in the negative feedback path of two power operational amplifiers PA12 (Apex Microtechnology Inc., Tucson, AZ). The output current was proportional to one single voltage input that was inverted in one channel to generate two opposite current outputs. An arbitrary current offset could be added to each channel independently to generate the alignment field. The maximum output current in each channel was 3.5 A. A voltage follower connected to the negative input of each operational amplifier allowed measuring the actual current value of each channel.

\section{Magnetizer}

Ferromagnetic beads can be typically magnetized to saturation by applying a magnetic field larger than 150 $\mathrm{mT}^{23,27}$ This field was produced by discharging a capacitor $(1 \mathrm{mF})$ into the pair of coils connected in series. Given that the coil inductance was $12.4 \mathrm{mH}$ and that their total resistance was $9.6 \Omega$, the resulting damping factor $(\xi=1.36)$ of the RLC discharging circuit ensured an overdamped response with no oscillations that could result in the demagnetization of the beads. The capacitor could be charged up to $300 \mathrm{~V}$ producing a field larger than $150 \mathrm{mT}$ during $10 \mathrm{~ms}$ with a maximum peak of $280 \mathrm{mT}(28 \mathrm{~A})$.

\section{Image acquisition and processing \\ 1. Image acquisition}

The microscope was placed on a vibration isolation table (Isostation, Newport, Irvine, CA). Images were acquired with a progressive scan black-and-white camera (CV-M10 BX, JAI, Denmark) with square pixels of $8.3 \mu \mathrm{m}$ side. The apparent pixel size after magnification $(40 \times)$ was $205 \mathrm{~nm}$ with a resulting field of view of $160 \mu \mathrm{m} \times 120 \mu \mathrm{m}$. The image acquisition timing was controlled with an external trigger with maximum delay of $2 \mu$ s between the active trigger edge and the start of an acquisition. The electronic shutter speed of the camera was set to $1 \mathrm{~ms}$ and could be increased to $0.1 \mathrm{~ms}$ to avoid blurred images. ${ }^{19}$ With this timing performance, oscillatory displacements up to $1000 \mathrm{~Hz}$ could be detected using heterodyne acquisition. ${ }^{28}$ The analog video signal was digitized and transferred to the PC memory by an 8-bit resolution frame grabber (PC Eye4, Eltec, Mainz, Germany).

\section{Image processing}

Image analysis was performed with an application developed in Visual $\mathrm{C}++$ 5.0. After a sequence of up to 300 full frames was stored in the RAM memory of a PC, a localizing algorithm scanned the first image in order to identify the 
beads. Since they appeared as dark objects on a gray background, any pixel below a user-defined threshold was initially assumed to belong to a bead. Next, a set of selection loops operating in accordance with user-defined parameters (bead size, shape and contrast, minimum distance between beads) was applied to a squared window of $N \times N$ pixels surrounding the previously identified beads. $N$ was selected according to the bead size, the minimum distance between two beads and the microscope magnification. After identifying any valid bead in the field of view, the centroid of each particle within the $N \times N$ window was computed as:

$$
X_{c m}=\frac{\sum_{k=1}^{N} \sum_{m=1}^{N} x_{k m}\left[I_{t h}-I(k, m)\right]}{\sum_{k=1}^{N} \sum_{m=1}^{N}\left[I_{t h}-I(k, m)\right]} \text { for } I_{t h}>I(k, m),
$$

where $X_{c m}$ is the coordinate of the centroid of the bead in the $x$ axis, $x_{k m}$ is the coordinate of each pixel in the $x$ axis, $I(k, m)$ is the pixel intensity, and $I_{t h}$ is an arbitrary threshold defined to separate the pixels that belong to the bead from the background. An equivalent equation was used to calculate the $y$ coordinate of the centroid. Once the beads were located in the first image, the tracking algorithm computed the position of each bead in the subsequent images. This was done by an iterative process that repositioned the squared window until the whole bead was centered on it.

After computing the position of each particle through the sequence, a moving average digital filter with a time window of one oscillation period was applied to the tracking signal. Data were filtered twice, forward and backward, with the same filter to eliminate phase shifts. The amplitude and phase of the bead oscillations were computed by Fourier analysis.

\section{Computer control}

Two different applications were developed to independently control the frame grabber and to drive a data acquisition and generation board (PCI-MIO-16XE-10, National Instruments, Austin, TX). The image application was written in Visual $\mathrm{C}++$ and operated as a slave of a LABVIEW software which controlled both the current fed to the coils and the external camera trigger by means of the two analog outputs of the data acquisition board. Whenever the master generated a trigger pulse, an image was acquired and digitized by the frame grabber while the actual current in each coil was simultaneously stored in a file.

\section{SYSTEM PERFORMANCE AND VALIDATION}

\section{A. Ferrimagnetic beads}

The designed magnetic tweezers system was validated by using ferrimagnetic beads $\left(\mathrm{Fe}_{3} \mathrm{O}_{4}\right)$ produced as described by Möller et al. ${ }^{29}$ and had a diameter of $4.5 \mu \mathrm{m} \pm 0.4 \mu \mathrm{m}$. The remanent magnetic moment of $200 \mu \mathrm{g}$ of beads was measured with a magnetic twisting rheometer, ${ }^{30}$ yielding $7.9 \times 10^{-13} \mathrm{Am}^{2}$ per bead.

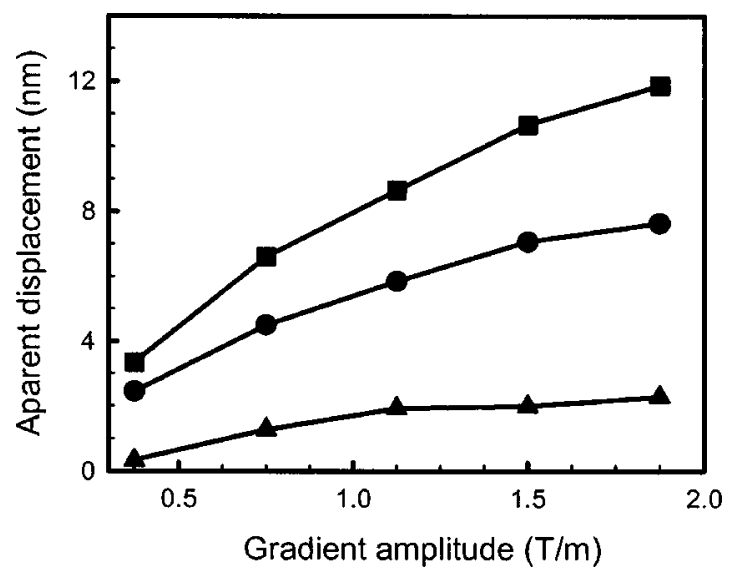

FIG. 5. Apparent bead displacement resulting from the magnetic force produced on the microscope objective. The three curves correspond to the oscillation amplitude in phase with the applied current for three different alignment fields: $0 \mathrm{mT}$ (triangles), $6 \mathrm{mT}$ (circles), and $12 \mathrm{mT}$ (squares).

\section{B. Magnetic coupling corrections}

The first step of the system validation consisted of a series of measurements on beads glued with epoxy in a $7 \mathrm{~mm}$ well. Bead tracking was performed during the application of an oscillatory $(1 \mathrm{~Hz})$ magnetic gradient of variable amplitude (0.4-1.9 mT) superimposed onto three different alignment fields $(0,6$, and $12 \mathrm{mT})$. Contrary to what was expected, we observed an oscillation in phase with the applied force (Fig. 5). The displacement amplitude increased with the applied magnetic gradient and alignment field. Only negligible changes $(5 \%)$ were observed when applying a series of magnetizing pulses before each measurement or when varying the frequency $(0.2-4 \mathrm{~Hz})$ of the applied gradient. The same results were obtained on magnetic and nonmagnetic immobile beads of different size, indicating that the oscillatory displacement detected was independent of the sample. Mechanical coupling was ruled out by mechanically isolating the coils from the microscope. Once the size and location of the coils with respect to the microscope objective were determined, the oscillation was reproducible.

These data suggest the generation of a magnetic pulling force on the microscope objective since it was the only weakly magnetic material in the setup. Such an artifact was not reported in previous magnetic tweezer designs even if higher gradients were produced. In subsequent measurements, the component due to magnetic coupling was subtracted from the actual data during the Fourier analysis.

\section{Tracking system resolution}

Tracking resolution was assessed by producing displacement oscillations $(0.2-200 \mathrm{~nm}, 2 \mathrm{~Hz})$ on fixed beads with a uniaxial piezotranslator (PA 16/14 SG, Piezosystem Jena, Germany) equipped with a position sensor. The system was controlled with a 16 bit A/D-D/A data acquisition board driven by LABVIEW software. The oscillatory signal feeding the piezotranslator was generated by the A/D-D/A board, low-pass filtered (Butterworth, eight poles, $8 \mathrm{~Hz}$ ) and amplified with a high voltage power operational amplifier (PA42, APEX, AZ). The sensor signal was amplified with a low- 


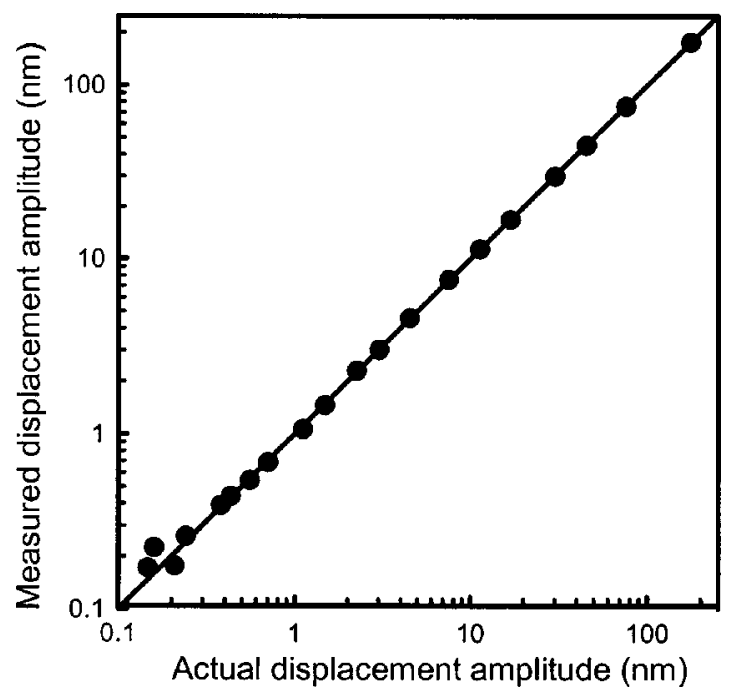

FIG. 6. Comparison between the actual oscillation amplitude of a bead (determined by the piezotranslator position sensor) and the oscillation amplitude measured by the tracking software. Image acquisition was performed on a single bead of $4.5 \mu \mathrm{m}$ in diameter for $50 \mathrm{~s}$ with a frame rate of $40 \mathrm{~Hz}$; solid line plots identity.

noise instrumentation amplifier (AMP-0, Precision Monolithics, CA), low-pass filtered (Butterworth analog filter, 8 $\mathrm{Hz}$, eight poles) and sampled at $5 \mathrm{kHz}$. For each oscillation amplitude, a sequence of 2000 images of a single bead was acquired at $40 \mathrm{~Hz}$. The amplitudes of both the sensor and the optical tracking signals were computed by Fourier analysis of the $50 \mathrm{~s}$ record. As shown in Fig. 6, the agreement between both signals reached the subnanometer range.

\section{Validation in a viscous standard}

\section{Spherical particle oscillating in a viscous fluid}

The performance of the whole experimental setup (magnetic actuator and tracking system) was evaluated by embedding the beads in a liquid of known viscosity and measuring their displacement in response to oscillatory excitations with various amplitudes and frequencies. The equation of motion for a bead of radius $a$, and magnetic moment $m$, subjected to a sinusoidal magnetic field of amplitude $B_{0 z}$ and frequency $\omega$ can be derived from Eq. (1) and from Stokes' law yielding:

$$
z(t)=-\frac{m}{6 \pi a \eta \omega} \frac{\partial B_{0 z}}{\partial z} \cos (\omega t),
$$

where $z$ is the position of the bead and $\eta$ is the fluid viscosity (the magnetic moment of the bead was assumed to be aligned with the applied field $B_{0 z}$ ). Therefore, the predicted response of the beads is an out of phase oscillation.

\section{Sample preparation}

The beads were dispersed in dymethilpolysiloxane (Sigma Chemical, St. Louis, MO) with a kinematic viscosity 500 cstk in low concentration to avoid particle-particle magnetic and hydrodynamic interactions. An amount of $200 \mu \mathrm{l}$ of the preparation was introduced into a $7 \mathrm{~mm}$ well. The beads were magnetized whenever a new field of view was

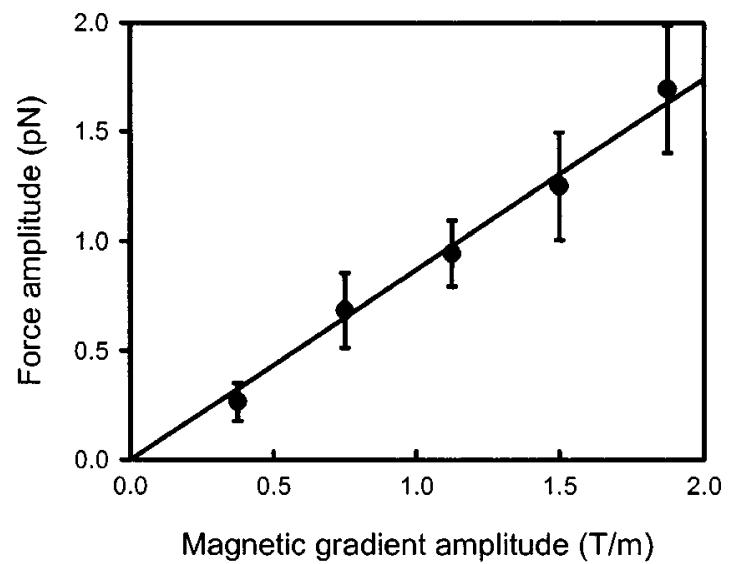

(a)

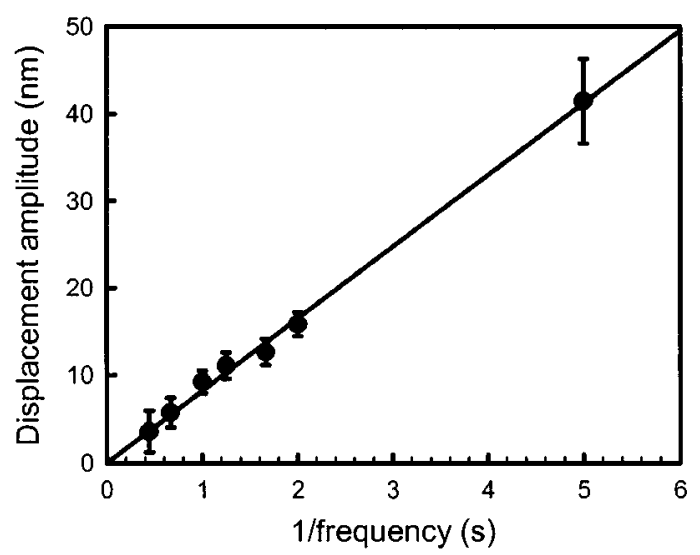

(b)

FIG. 7. (a) Amplitude of the magnetic force acting on the beads (mean $+\mathrm{SD}, n=4)$ as a function of the amplitude of the applied magnetic gradient at a frequency of $0.5 \mathrm{~Hz}$; (b) frequency dependence of the out of phase component of the bead displacement (mean $+\mathrm{SD}, n=3$ ) at a constant gradient amplitude of $1.1 \mathrm{~T} / \mathrm{m}$.

selected. Only the beads that had a circular cross-sectional appearance and were at least ten bead diameters apart from other beads were selected for measurements.

\section{Amplitude and frequency response}

The experimental setup was validated through two series of measurements varying both the amplitude and the frequency of the applied magnetic gradient. Figure 7(a) displays the measured force amplitude as a function of the applied magnetic gradient $(0.5 \mathrm{~Hz})$. The force was derived from Stokes' law and from the out of phase component of the measured bead displacement $(n=4)$. As expected, the force increased linearly with the gradient reaching $1.7 \mathrm{pN}$ for a gradient of $1.9 \mathrm{~T} / \mathrm{m}$. Figure 7(b) shows the out of phase component of the displacement as a function of the oscillation period $(f=0.2-2.25 \mathrm{~Hz})$ for a constant gradient of 1.1 $\mathrm{T} / \mathrm{m}(n=3)$. According to the behavior predicted by Stokes' law, a linear relationship was found. By using Eq. (12), the magnetic moment of the beads was derived from the slopes of the curves in Fig. 7, yielding $8.7 \times 10^{-13} \mathrm{~A} \mathrm{~m}^{2}$ from the force curve and $8.2 \times 10^{-13} \mathrm{~A} \mathrm{~m}^{2}$ from the frequency curve. The discrepancy between these values and the value measured with the magnetic twisting rheometer was $<10 \%$. 


\section{DISCUSSION}

In this work we designed and validated a simple device to apply oscillatory uniaxial forces on micronsized ferromagnetic beads. The method was based on two subsequent steps, magnetization and application of the force, which allowed us to keep the magnetic moment of the beads constant and known throughout the experiments. A single pair of coils was optimized to produce a magnetizing pulse up to $\sim 280 \mathrm{mT}$ in direct configuration and a pulling magnetic gradient up to $\sim 2 \mathrm{~T} / \mathrm{m}$ in inverse configuration, both with a variation lower than $5 \%$ within the sample. We validated the system with 4.5 $\mu \mathrm{m}$ ferrimagnetic beads obtaining forces up to $\sim 2 \mathrm{pN}$ and subnanometer tracking resolution.

\section{A. Magnetic field optimization}

We optimized a pair of pure inductances to produce high fields and gradients without using soft magnetic cores that would restrict the bandwidth and require feedback to achieve stable and reproducible forces. ${ }^{21}$ The optimization of the shape, dimensions and separation of a pair of coaxial coils has been largely studied for a wide range of applications. ${ }^{31-34}$ These designs typically focus on maximizing the field $^{35,36}$ or the gradient ${ }^{24}$ homogeneity either in the direct or the inverse configurations. Accordingly, such implementations use the classical Helmholtz $(\alpha=1)$ or Maxwell $(\alpha$ $=0.58$ ) pairs that ensure the highest homogeneity of the field or of the gradient, respectively. However, none of these configurations produce the highest fields or gradients achievable, as shown in Fig. 2. By contrast, we optimized the coil design to work in both configurations simultaneously, ensuring high and homogeneous fields and gradients. We chose a nearMaxwell configuration that allowed us to permanently magnetize, pull and align ferromagnetic beads by creating large fields and gradients with a variation lower than $5 \%$ over the sample area.

\section{B. Ferromagnetic beads}

Most of the magnetic tweezers reported to date use superparamagnetic beads. ${ }^{20-22}$ These beads are homogeneous in size and shape and do not show any remanent magnetization after removal of a magnetizing field. However, their magnetic moment is low and, consequently, relatively high gradients are required to reach forces in the $\mathrm{pN}$ range. In addition, as their magnetic moment nonlinearly depends on the applied magnetizing field, the relationship between the applied gradient and the resulting force is nonlinear unless the beads are saturated. Owing to this nonlinearity, when the coils are fed with an oscillatory current, the force generated on the beads is only approximately sinusoidal. ${ }^{19}$

To overcome these limitations, our device uses permanently magnetized ferromagnetic beads. These beads can be subjected to a secondary magnetization in a weak magnetic field without rotation of magnetic domains, i.e., with no change in their magnetic moment. ${ }^{23}$ This property of ferromagnetic (and ferrimagnetic) materials allowed the measurement of the mechanical properties of viscous solutions, ${ }^{37}$ intracellular organelles, ${ }^{38}$ and cytoskeleton ${ }^{6,28,39}$ by twisting beads through a weak magnetic field in a direction different from that of the bead magnetic moment. Instead of applying a twisting torque, we used permanently magnetized beads to produce controlled translational forces. As the field produced in the central region between the coils was low, the magnetic moment of the beads remained constant throughout the experiments. This was shown by the linear relationship found between the current in the coils and the force generated on the beads [Fig. 7(a)]. Given that the magnetic gradient was analytically derived and that the remanent magnetic moment of the beads was known, the force on the sample could be computed as a linear function of the current in the coils. Hence, unlike other magnetic tweezers, no calibration of the system is required.

The values of the remanent magnetic moment of the beads obtained with the magnetic tweezers were slightly higher than the value measured with the magnetic twisting rheometer. This discrepancy could be explained by the fact that magnetic twisting rheometry computes the average remanent magnetic moment of a large population of beads. Consequently, the results can be biased by the presence of a small fraction of nonspherical particles or by the formation of clusters of beads in the preparation. ${ }^{23,30}$ By contrast, magnetic tweezers allow the measurement of individual beads providing a more accurate estimate of their remanent magnetic moment.

A limitation of using magnetized ferromagnetic beads could arise from their tendency to align with the applied magnetic field, which is not necessarily aligned in the axial direction. To overcome this potential limitation we superimposed a constant and weak magnetic field aligned with the coil axis. This alignment field prevented the beads from pivoting and reduced the twisting angle to $5^{\circ}$ over the central circle (1 mm wide).

\section{Image resolution}

The position of an object and its relative displacements can be measured with nanometer resolution by using videomicroscopy-based tracking algorithms. ${ }^{40-42}$ Recently, a quantitative simulation analysis pointed out the limitations of the most frequently used particle tracking algorithms under conditions of low signal-to-noise ratio. ${ }^{43}$ The resolution of these algorithms is currently assessed by tracking the position of a fixed particle and computing the standard deviation of the resulting displacement. This technique accounts for the main sources of noise of the system and provides an estimate of the lowest displacement that can be detected. However, to the best of our knowledge, no experimental data assessing the resolution of the algorithms for displacements close to the noise boundary have been reported. We used a piezotranslator to produce controlled displacements on stationary beads in conditions of low signal-to-noise ratio $(0.1-$ 100). This method, previously used to calibrate laser tracking systems, ${ }^{44,45}$ allowed us to compare the measurement of bead displacement and its actual displacement. Although the standard deviation of a single stationary bead $(4.5 \mu \mathrm{m})$ was 1.2 $\mathrm{nm}$, near-Angstrom oscillatory displacements were accurately determined by computing the FFT of the optical tracking signal. 


\section{Applicability and improvements}

The apparatus described in this work is suitable for use in a wide range of experiments that require the application of oscillatory uniaxial forces in the piconewton scale while sensing the sample response with nanometer resolution. This range of forces and displacements is suitable for microrheological measurements of biopolymer networks, such as F-actin, ${ }^{3,19,46}$ collagen, ${ }^{47}$ or filamentous viruses. ${ }^{48}$ Typical values of the shear modulus of these solutions range from 0.01 to $100 \mathrm{~Pa}$. According to Ziemann and co-workers, ${ }^{4}$ an oscillatory bead rheometer with a force of $2 \mathrm{pN}$ and a resolution of $1 \mathrm{~nm}$ in the detection of the bead amplitude can estimate the shear modulus of solutions up to $\sim 50 \mathrm{~Pa}$. Our device also features the range of forces and displacements required to manipulate macromolecules. ${ }^{1,2,20,49}$ Typically, in these measurements the signal corresponding to a single probe is acquired in each experiment. As our system produces a homogeneous force field and allows multiple particle tracking, the properties of a large number of macromolecules could be measured in parallel, reducing drastically the number of experiments required to obtain statistical results. ${ }^{17}$ Magnetic actuators in the piconewton range have also been used to probe the mechanotransduction in living cells by specifically binding magnetic beads to transmembrane receptors. ${ }^{6}$ Goldschmit et al. demonstrated that living cells are capable of sensing cyclic forces in the piconewton range. ${ }^{50}$ They used a device that applied forces $(0.2 \mathrm{pN}$ per bead) normal to the cell surface on ferromagnetic beads in the range $0.01-2 \mathrm{~Hz}$. The device described in this article can apply a wide range of shear forces and could allow the measurement of cell mechanotransduction over a wide frequency band.

Modifying the magnitude of the force applied may allow us to extend the applications of the designed magnetic tweezers. Increasing the force applied could be of interest in micro- and nanomanipulation experiments of biological relevance such as measuring the strength of receptor-ligand bonds ${ }^{51,52}$ or probing the mechanical properties of the cytoskeleton. ${ }^{7,53}$ Such forces could be reached by increasing the amplitude of the magnetic gradient. Since the coils cannot be brought closer, the gradient could only be enhanced by increasing the number of turns per coil or the current. In both cases, a substantial improvement in the applied force would lead to a high raise in the power dissipation and to the need for more elaborated cooling systems like watercooling jackets. ${ }^{20}$ Moreover, even if the field always vanishes in the central plane between the coils, it could attain high values within the sample and produce a noncontrolled remagnetization of the beads. Therefore, their magnetic moment would no longer be constant and known throughout the experiments. A more feasible option to increase the amplitude of the force applied with the described apparatus would be to increase the remanent magnetic moment of the beads. This could be done by simply using larger beads. As the magnetic moment of a bead is proportional to its volume, a twofold increase in the radius would result in an improvement of almost one order of magnitude in force. Increasing the remanent magnetic field of the beads could also be achieved by using stronger magnetic materials. ${ }^{54}$ As regards the potential use of the designed magnetic tweezers in applications requiring low force generation, it is interesting to note that the device described could be particularly suitable given the analytical relationship between current and force. Indeed, well controlled small forces can be accurately applied by simply reducing the driving current. This contrasts with other techniques such as laser tweezers where the determination of the applied force is affected by the experimental noise since force is indirectly measured from bead displacement. ${ }^{12}$

\section{ACKNOWLEDGMENTS}

The authors wish to thank D. Navajas, M. Rotger, and V. F. Muntes for their helpful comments and suggestions and Miguel Rodriguez for his technical assistance. This work was supported in part by grants from Ministerio de Ciencia y Tecnologia (SAF 2002-03616), NIH-HL65960, and by Ministerio de Sanidad y Consumo (Red GIRA, G03/063).

${ }^{1}$ S. B. Smith, L. Finzi, and C. Bustamante, Science 258, 1122 (1992).

${ }^{2}$ D. Wirtz, Phys. Rev. Lett. 75, 2436 (1995).

${ }^{3}$ F. Amblard, A. C. Maggs, B. Yurke, A. Pargellis, and S. Leibler, Phys. Rev. Lett. 77, 4470 (1996).

${ }^{4}$ F. Ziemann, J. Radler, and E. Sackmann, Biophys. J. 66, 2210 (1994).

${ }^{5}$ F. Crick and A. F. W. Hugues, Exp. Cell Res. 1, 37 (1950).

${ }^{6}$ N. Wang, J. P. Butler, and D. E. Ingber, Science 260, 1124 (1993).

${ }^{7}$ A. R. Bausch, W. Moller, and E. Sackmann, Biophys. J. 76, 573 (1999).

${ }^{8}$ B. Fabry, G. N. Maksym, J. P. Butler, M. Glogauer, D. Navajas, and J. J. Fredberg, Phys. Rev. Lett. 87, 148102 (2001).

${ }^{9}$ C. Bustamante, J. C. Macosko, and G. J. Wuite, Nat. Rev. Mol. Cell Biol. 1, 130 (2000).

${ }^{10}$ G. Binnig, C. F. Quate, and C. Gerber, Phys. Rev. Lett. 56, 930 (1986).

${ }^{11}$ A. Ashkin and J. M. Dziedzic, Phys. Rev. Lett. 54, 1245 (1985).

${ }^{12}$ K. Svoboda and S. M. Block, Annu. Rev. Biophys. Biomol. Struct. 23, 247 (1994).

${ }^{13}$ A. Kishino and T. Yanagida, Nature (London) 334, 74 (1988).

${ }^{14}$ Y. Liu, G. J. Sonek, M. W. Berns, and B. J. Tromberg, Biophys. J. 71, 2158 (1996).

${ }^{15}$ K. C. Neuman, E. H. Chadd, G. F. Liou, K. Bergman, and S. M. Block, Biophys. J. 77, 2856 (1999).

${ }^{16}$ T. R. Strick, J. F. Allemand, D. Bensimon, A. Bensimon, and V. Croquette, Science 271, 1835 (1996).

${ }^{17}$ F. Assi, R. Jenkins, J. Yang, C. Love, and M. Prentiss, J. Appl. Phys. 92, 5584 (2002).

${ }^{18}$ F. G. Schmidt, F. Ziemann, and E. Sackmann, Eur. Biophys. J. 24, 348 (1996).

${ }^{19}$ M. Keller, J. Schilling, and E. Sackmann, Rev. Sci. Instrum. 72, 3626 (2001).

${ }^{20}$ C. Haber and D. Wirtz, Rev. Sci. Instrum. 71, 4561 (2000).

${ }^{21}$ F. Amblard, B. Yurke, A. Pargellis, and S. Leibler, Rev. Sci. Instrum. 67, 818 (1996).

${ }^{22}$ C. Gosse and V. Croquette, Biophys. J. 82, 3314 (2002).

${ }^{23}$ P. A. Valberg and J. P. Butler, Biophys. J. 52, 537 (1987).

${ }^{24}$ P. N. Murgatroyd and B. E. Bernard, Rev. Sci. Instrum. 54, 1736 (1983).

${ }^{25}$ J. D. Jackson, Classical Electrodynamics (Wiley, New York, 1975).

${ }^{26}$ P. N. Murgatroyd, Am. J. Phys. 59, 949 (1991).

${ }^{27}$ W. Moller, G. Scheuch, K. Sommerer, and J. Heyder, J. Magn. Magn. Mater. 225, 8 (2001).

${ }^{28}$ B. Fabry, G. N. Maksym, S. A. Shore, P. E. Moore, R. A. J. Panettieri, J. P. Butler, and J. J. Fredberg, J. Appl. Physiol. 91, 986 (2001).

${ }^{29}$ W. Moller, C. Roth, and W. Stahlhofen, J. Aerosol Sci. 21, S657 (1990).

${ }^{30}$ K. S. Zaner and P. A. Valberg, J. Cell Biol. 109, 2233 (1989).

${ }^{31}$ M. Rudd and J. Craig, Rev. Sci. Instrum. 39, 1372 (1968).

${ }^{32}$ R. Cacak and J. Craig, Rev. Sci. Instrum. 40, 1468 (1969).

${ }^{33}$ K. Kaminishi and S. Nawata, Rev. Sci. Instrum. 52, 447 (1981).

${ }^{34}$ J. Wang, S. She, and S. Zhang, Rev. Sci. Instrum. 73, 2175 (2002).

${ }^{35}$ M. A. Nordahn, T. Holst, and Y. Q. Shen, Supercond. Sci. Technol. 12, 946 (1999).

${ }^{36}$ R. A. J. Schill and K. Hoff, Rev. Sci. Instrum. 72, 2769 (2001). 
${ }^{37}$ W. Moller, W. Stahlhofen, and J. Wiegand, J. Aerosol Sci. 23, S421 (1992).

${ }^{38}$ W. Moller, I. Nemoto, T. Matsuzaki, T. Hofer, and J. Heyder, Biophys. J. 79, 720 (2000).

${ }^{39}$ G. N. Maksym, B. Fabry, J. P. Butler, D. Navajas, D. J. Tschumperlin, J. D. Laporte, and J. J. Fredberg, J. Appl. Physiol. 89, 1619 (2000).

${ }^{40}$ J. Gelles, B. J. Schnapp, and M. P. Sheetz, Nature (London) 331, 450 (1988).

${ }^{41}$ S. Inoué, Methods Cell Biol. 30, 85 (1989).

${ }^{42}$ M. J. Saxton and K. Jacobson, Annu. Rev. Biophys. Biomol. Struct. 26, 399 (1997)

${ }^{43}$ M. K. Cheezum, W. F. Walker, and W. H. Guilford, Biophys. J. 81, 2378 (2001).

${ }^{44}$ F. Gittes, B. Schnurr, P. D. Olmsted, F. C. MacKintosh, and C. F. Schmidt, Phys. Rev. Lett. 79, 3286 (1997).

${ }^{45}$ S. Yamada, D. Wirtz, and S. C. Kuo, Biophys. J. 78, 1736 (2000).
${ }^{46}$ F. G. Schmidt, B. Hinner, and E. Sackmann, Phys. Rev. E 61, 5646 (2000).

${ }^{47}$ D. Velegol and F. Lanni, Biophys. J. 81, 1786 (2001).

${ }^{48}$ F. G. Schmidt, B. Hinner, E. Sackmann, and J. X. Tang, Phys. Rev. E 62 , 5509 (2000)

${ }^{49}$ T. R. Strick, V. Croquette, and D. Bensimon, Nature (London) 404, 901 (2000).

${ }^{50}$ M. E. Goldschmidt, K. J. McLeod, and W. R. Taylor, Circ. Res. 88, 674 (2001).

${ }^{51}$ E. L. Florin, V. T. Moy, and H. E. Gaub, Science 264, 415 (1994).

${ }^{52}$ R. Merkel, P. Nassoy, A. Leung, K. Ritchie, and E. Evans, Nature (London) 397, 50 (1999).

${ }^{53}$ F. J. Alenghat, B. Fabry, K. Y. Tsai, W. H. Goldmann, and D. E. Ingber, Biochem. Biophys. Res. Commun. 277, 93 (2000).

${ }^{54}$ C. Abdelghani-Jacquin, A. Abdelghani, G. Chmel, M. Kantlehner, and E. Sackmann, Eur. Biophys. J. 31, 102 (2002). 